TUM-HEP 722/09

\title{
Gauge-top unification
}

\author{
Pierre Hosteinst, Rolf Kappl2, Michael Ratz], Kai Schmidt-Hoberg 4 \\ Physik-Department T30, Technische Universität München, \\ James-Franck-Straße, 85748 Garching, Germany
}

\begin{abstract}
Higher-dimensional models of grand unification allow us to relate the top Yukawa coupling $y_{t}$ to the gauge coupling $g$. The tree level relation $y_{t}=g$ at the scale of grand unification implies, in the framework of the MSSM, a rather small ratio of Higgs expectation values $\tan \beta$. We find that, in the presence of localized Fayet-Iliopoulos terms, $y_{t}$ is suppressed against $g$ because the bulk fields acquire non-trivial profiles whose overlap is smaller than in the case of flat profiles. This increases the prediction for $\tan \beta$ to moderately large values. Thus $\tan \beta$ is related to the geometry of compact space. We also discuss explicit realizations of such settings in orbifold compactifications of the heterotic string. It turns out that anisotropic compactifications, allowing for an orbifold GUT interpretation, are favored.
\end{abstract}

\footnotetext{
${ }^{1}$ Email: pierre.hosteins@ph.tum.de

${ }^{2}$ Email: rolf .kappl@ph.tum.de

${ }^{3}$ Email: mratz@ph.tum.de

${ }^{4}$ Email: kschmidt@ph.tum.de
} 


\section{Introduction}

The coupling strengths governing the interactions of the standard model (SM) exhibit a very peculiar pattern: on the one hand, the gauge and top Yukawa couplings are of order one, on the other hand all other Yukawa couplings are suppressed. This might tell us that couplings come in two classes with fundamentally different origin. In this study we shall investigate the scenario of "gauge-top unification" in which the top Yukawa coupling $y_{t}$ and the gauge couplings $g_{a}(1 \leq a \leq 3)$ unify at a high scale. Our analysis will be based on the minimal supersymmetric extension of the standard model (MSSM), as it appears to provide us with the most compelling scenario of gauge coupling unification, and therefore fits very nicely to the concept of grand unified theories (GUTs) [1,2].

Arguably, the most compelling realizations of GUTs incorporate extra dimensions. In fact, string-theoretic orbifolds [3-10] and field-theoretic orbifold GUTs [11-18] allow us to retain the beautiful aspects of grand unification while avoiding the notorious problems. Merging both approaches [19-22] has lead us to explicit string-derived models which reproduce the MSSM in their low-energy limit and have a straightforward orbifold GUT interpretation [23-28] (for reviews see [29,30]).

In this paper we shall study scenarios in which $y_{t}$ arises from gauge interactions in more than four dimensions. We will discuss settings in which the Higgs fields arise from extra components of the gauge multiplet, enforcing the tree-level relation

$$
y_{t}=g
$$

at the compactification scale [18,31]. As we shall see, relation (1) implies, together with the updated mass of the top quark [32], uncomfortably small values for the ratio of Higgs expectation values $\tan \beta$. However, following earlier work by Lee, Nilles and Zucker [33] we find that localized Fayet-Iliopoulos terms, which are generically present in these compactifications, always reduce the value of $y_{t}$ at the compactification scale, thus increasing the prediction for $\tan \beta$ to moderately large (or even large) values. This is because the bulk hypermultiplets attain non-trivial profiles whose overlap is always smaller than in the case of flat profiles. The precise value of $\tan \beta$ depends on the size and shape of compact space.

This paper is organized as follows. In section 2 we will discuss a class of simple orbifold GUT models in which $y_{t}=g$ at tree level. Section 3 is devoted to (quantum) corrections to this relation. In section 4 we will discuss explicit string theory realizations of these settings. Phenomenological implications are described in section 5. Finally, section 6 contains our conclusions. 


\section{Gauge-top unification in extra dimensions}

Let us now consider field-theoretic orbifold GUT settings in which $y_{t}$ arises from gauge interactions. We shall focus on models with two extra dimensions and an SU(6) bulk gauge group. We will also consider further U(1) factors, as their presence has important implications for the prediction of gauge and Yukawa couplings. The geometry of the model is $\mathbb{T}^{2} / \mathbb{Z}_{2}$. The resulting orbifold can be envisaged as a ravioli or pillow, whose corners correspond to the four fixed points. We label the fixed points by two integers $n_{2}$ and $n_{2}^{\prime}$ which take the values 0 or 1 (see figure 1). The lengths of the edges of the pillow or the distances of the fixed points are given by $\pi R_{5}$ and $\pi R_{6}$, respectively, where $R_{5}$ and $R_{6}$ denote the radii of the underlying 2 -torus $\mathbb{T}^{2}$. We restrict ourselves to rectangular tori, leaving the general case for future analysis.

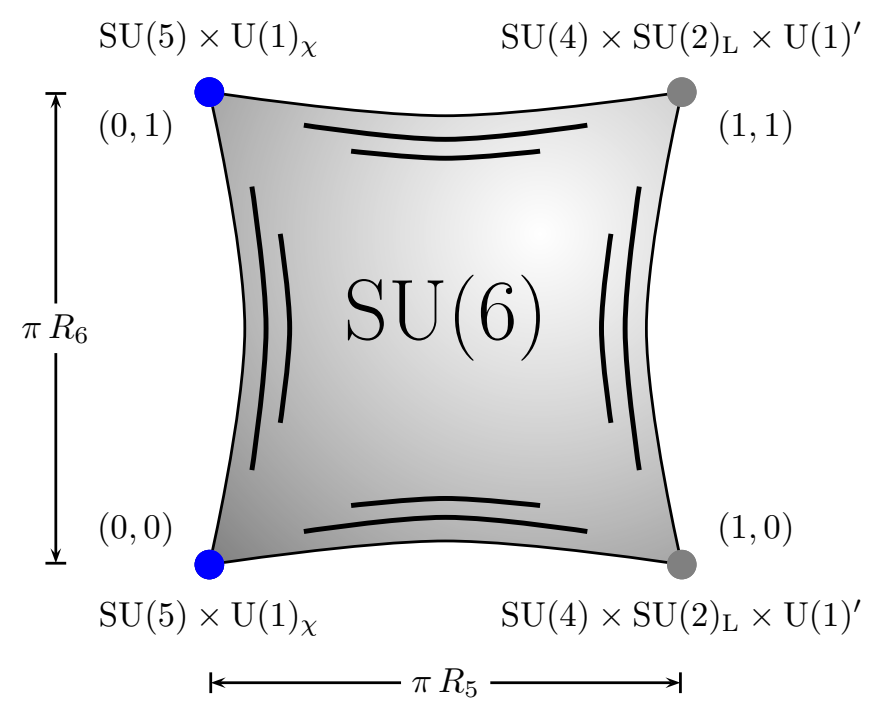

Figure 1: 6D orbifold GUT. We show the local gauge groups at the fixed points, labeled by the localization quantum numbers $\left(n_{2}, n_{2}^{\prime}\right)$.

The $\mathrm{SU}(6)$ bulk gauge group gets broken to $\mathrm{SU}(5) \times \mathrm{U}(1)_{\chi}$ and $\mathrm{SU}(4) \times \mathrm{SU}(2)_{\mathrm{L}} \times \mathrm{U}(1)^{\prime}$ at two inequivalent fixed points, i.e. fixed points with different $n_{2}$. The low-energy gauge group emerges as the intersection of these local gauge groups, and is given by

$$
G_{\mathrm{SM}}=\mathrm{SU}(3)_{C} \times \mathrm{SU}(2)_{\mathrm{L}} \times \mathrm{U}(1)_{Y}
$$

plus an additional U(1) factor. In orbifold GUT language, this is a consequence of the $\mathbb{Z}_{2}$ boundary conditions

$$
P=\operatorname{diag}(1,1,1,1,1,-1) \text { and } P^{\prime}=\operatorname{diag}(1,1,1,-1,-1,1),
$$

which are to be imposed at the fixed points with $n_{2}=0$ and $n_{2}=1$, respectively. In the description common to string theory, the difference between the two local boundary conditions can be ascribed to the presence of a discrete order 2 Wilson line in $y_{5}$ direction. The emerging symmetry breaking pattern has been studied in the context of a $5 \mathrm{D}$ 
orbifold GUT [18], and happens to arise in a 6D orbifold GUT limit of promising string compactifications $[26,29,30]$. The bulk supersymmetry in six dimensions corresponds to $N=2$ supersymmetry from a $4 \mathrm{D}$ perspective. The $6 \mathrm{D}$ gauge multiplet $(V, \Phi)$ contains the $4 \mathrm{D}$ vector field $V$ as well as the chiral field $\Phi$. The boundary conditions (3) result in orbifold parities for the components of $\Phi$ that are opposite to those of $V$,

$$
\begin{aligned}
\Phi & =\Phi^{a} \top_{a} \\
& =\left(\begin{array}{ccc}
\Phi_{(\mathbf{8}, \mathbf{1})_{0}}^{(-)}-\frac{1}{\sqrt{15}} \Phi_{Y}^{(--)}+\frac{1}{2 \sqrt{15}} \Phi_{\chi}^{(--)} & \frac{1}{\sqrt{2}} \Phi_{(\mathbf{3 , 2})_{-5 / 6}}^{(-+)} & \frac{1}{\sqrt{2}} \Phi_{(\mathbf{3}, \mathbf{1})_{-1 / 3}}^{(+-)} \\
\frac{1}{\sqrt{2}} \Phi_{(\overline{\mathbf{3}}, \mathbf{2})_{5 / 6}}^{(-)} & \Phi_{(\mathbf{1}, \mathbf{3})}^{(-)}+\frac{3}{2 \sqrt{15}} \Phi_{Y}^{(--)}+\frac{1}{2 \sqrt{15}} \Phi_{\chi}^{(--)} & \frac{1}{\sqrt{2}} \Phi_{(\mathbf{1}, \mathbf{2})_{1 / 2}}^{(+)} \\
\frac{1}{\sqrt{2}} \Phi_{(\overline{\mathbf{3}}, \mathbf{1})_{1 / 3}}^{(-)} & \frac{1}{\sqrt{2}} \Phi_{(\mathbf{1}, \mathbf{2})_{-1 / 2}}^{(++)} & \frac{-5}{2 \sqrt{15}} \Phi_{(\mathbf{1}, \mathbf{1})_{0}}^{(-)}
\end{array}\right) .
\end{aligned}
$$

Here we decompose the generators of $\mathrm{SU}(6)$ into the generators of $G_{\mathrm{SM}} \times \mathrm{U}(1)_{\chi}$ and elements of the coset, using an obvious notation. At the massless level, only the doubly even states

$$
h_{u}=\Phi_{(\mathbf{1 , 2})_{1 / 2}}^{(+)} \quad \text { and } \quad h_{d}=\Phi_{(\mathbf{1 , 2})_{-1 / 2}}^{(++)}
$$

are retained, which carry the quantum numbers of the MSSM Higgs doublets. The normalization factors in (4) are a consequence of the usual condition on the generators,

$$
\operatorname{tr}\left(\mathrm{T}_{a} \mathrm{~T}_{b}\right)=\frac{1}{2} \delta_{a b}
$$

Both $\Phi=\Phi^{a} T_{a}$ and the various multiplets appearing on the right-hand side of (4) are canonically normalized.

The settings further contain a bulk hypermultiplet $H$ that transforms as a 20-plet under $\mathrm{SU}(6)$. In $N=1$ language

$$
H=\left(\varphi, \varphi^{c}\right)
$$

where $\varphi$ transforms as $\mathbf{2 0}$ (3-index antisymmetric tensor) and $\varphi^{c}$ as $\overline{\mathbf{2 0}}$ under SU(6). After orbifold projection, $\varphi$ gives rise to one copy of quark doublets $\left(q_{3}\right)$ and $\varphi^{c}$ leads to one superfield transforming as a $u$-type quark $\left(\bar{u}_{3}\right)$, as well as a lepton singlet $\bar{e}_{3}$. We refrain from specifying the origin of the remaining SM matter here. Later, in section 4, we will clarify this issue in the context of string-derived models, where all anomaly constraints are automatically fulfilled.

In order to extract the top Yukawa coupling it is sufficient to work with component fields. We will then denote by $\xi$ and $\eta$ the two-component spinors contained respectively in $\varphi$ and $\varphi^{c}$, and the scalar component of $\Phi$ is given by

$$
\phi=\frac{A_{5}+\mathrm{i} A_{6}}{\sqrt{2}} .
$$

The 4D Yukawa term

$$
y_{t} \bar{u}_{3} q_{3} h_{u} \subset \mathscr{L}_{4 \mathrm{D}}
$$


originates from the gauge interactions

$$
\eta\left(\bar{\partial}+\sqrt{2} g_{6} \phi\right) \xi \subset \mathscr{L}_{6 \mathrm{D}}
$$

with $g_{6}$ denoting the $6 \mathrm{D}$ gauge coupling and $\bar{\partial}=\partial_{5}+\mathrm{i} \partial_{6}$ (cf. [34]).

In $\mathrm{SU}(n)$ notation (see e.g. [35]), one has specifically for the $\mathrm{SU}(6)$ case under consideration

$$
\begin{aligned}
& \frac{1}{3 !} \eta_{i_{1} i_{2} i_{3}}\left[\delta_{j_{1}}^{i_{1}} \delta_{j_{2}}^{i_{2}} \delta_{j_{3}}^{i_{3}} \bar{\partial}+\sqrt{2} g_{6}\left(\phi_{j_{1}}^{i_{1}} \delta_{j_{2}}^{i_{2}} \delta_{j_{3}}^{i_{3}}+\delta_{j_{1}}^{i_{1}} \phi_{j_{2}}^{i_{2}} \delta_{j_{3}}^{i_{3}}+\delta_{j_{1}}^{i_{1}} \delta_{j_{2}}^{i_{2}} \phi_{j_{3}}^{i_{3}}\right)\right] \xi^{j_{1} j_{2} j_{3}} \\
& \quad=\frac{1}{3 !} \eta_{i_{1} i_{2} i_{3}}\left[\delta_{j_{3}}^{i_{3}} \bar{\partial}+3 \sqrt{2} g_{6} \phi_{j_{3}}^{i_{3}}\right] \xi^{i_{1} i_{2} i_{3}} .
\end{aligned}
$$

$q_{3}$ is contained in the $\mathrm{SU}(5)$ 10-plet from the $\mathrm{SU}(6)$ 20-plet $\xi$,

$$
\mathbf{1 0}^{i j}=\xi^{i j 6}
$$

while $\bar{u}_{3}$ arises from the $\mathbf{1 0}$-plet in the $\mathrm{SU}(6) \overline{\mathbf{2 0}}$-plet $\eta$,

$$
\frac{1}{2} \varepsilon_{i j k \ell m}\left(\mathbf{1 0}^{c}\right)^{\ell m}=\eta_{i j k}
$$

Here the indices $i, j, k \ldots$ run from 1 to 5 ; the above implies that $q_{3}$ and $\bar{u}_{3}$ do not stem from the same $\mathrm{SU}(5)$ 10-plet.

We are now left with

$$
\begin{aligned}
\sqrt{2} g_{6} \frac{1}{2} \eta_{i j k} \phi_{6}^{k} \xi^{i j 6} & =\frac{\sqrt{2} g_{6}}{4} \varepsilon_{i j k \ell m}\left(\mathbf{1 0}^{c}\right)^{i j} \mathbf{1 0}^{k \ell} \phi_{6}^{m} \\
& \supset \frac{g_{6}}{2} \varepsilon_{a b c} \varepsilon_{\alpha \beta} \varepsilon^{a b d}\left(\bar{u}_{3}\right)_{d}\left(q_{3}\right)^{c \alpha}\left(h_{u}\right)^{\beta} \\
& =g_{6} \bar{u}_{3} q_{3} h_{u} .
\end{aligned}
$$

Here $a, b, c, d$ are $\mathrm{SU}(3)_{C}$ indices and $\alpha, \beta$ are $\mathrm{SU}(2)_{\mathrm{L}}$ indices. This calculation shows that $y_{t}=g$ at tree level, and confirms an earlier computation [26] (see also [18]).

\section{Corrections to the equality of $y_{t}$ and $g$}

Having seen that $y_{t}=g$ at tree level, we now turn to discussing corrections to this relation. Such corrections are of different origin. First, there are radiative, logarithmic contributions to the wave function renormalization constants coming from fields localized at the fixed points (or 'branes') which do not respect the unified bulk gauge symmetry. Second, the top Yukawa coupling emerges from the $3 \times 3$ Yukawa matrix $Y_{u}$ by diagonalization. Subdominant entries in $Y_{u}$ can therefore shift this eigenvalue. Third, there are corrections coming from non-trivial localization properties of the bulk hypermultiplets. As we shall see, this effect yields generically the numerically dominant correction. 


\subsection{Corrections from localized states}

The $6 \mathrm{D} \beta$-function is comprised of a 'power-law' and logarithmic piece,

$$
\beta_{6 \mathrm{D}}=b_{6} \mu^{2} R_{5} R_{6}+b_{4}
$$

We have verified that the power-law pieces are universal for gauge and top couplings, as it should be. Assuming only the minimal matter content in the bulk to form the third family of the MSSM, one obtains

$$
b_{6}^{t}=b_{6}^{i}=-4 \text { for } 1 \leq i \leq 3 .
$$

The logarithmic corrections are sensitive to fields sitting at the fixed points, where the $\mathrm{SU}(6)$ bulk symmetry is broken. The $b_{4}$ coefficients might hence not be universal. One might think of these corrections as wave function renormalization constants localized at the fixed points. Their impact can be estimated as

$$
\left|\Delta y_{t}-\Delta g_{i}\right|_{\log } \sim\left|\Delta g_{i}-\Delta g_{j}\right|_{\log } \sim\left|\Delta b_{4} \ln \left(\Lambda / M_{\mathrm{GUT}}\right)\right|
$$

where $\Lambda$ denotes the cut-off and $\Delta b_{4}$ denotes the respective difference of $\beta$-functions. These corrections are expected to be numerically similarly relevant as MSSM threshold corrections, which originate from the squarks and sleptons having masses that differ by an $\mathcal{O}(1-10)$ factor. Such effects will not be studied in detail in the present analysis.

\subsection{Diagonalization effects}

In general, we will expect the $u$-type Yukawa matrix to be of the form

$$
Y_{u}=\left(\begin{array}{ccc}
0 & 0 & 0 \\
0 & 0 & 0 \\
0 & 0 & \mathcal{O}(g)
\end{array}\right)+\left(\begin{array}{ccc}
s^{n_{11}} & s^{n_{12}} & s^{n_{13}} \\
s^{n_{21}} & s^{n_{22}} & s^{n_{23}} \\
s^{n_{31}} & s^{n_{32}} & s^{n_{33}}
\end{array}\right),
$$

where $s$ denotes the typical expectation value of some singlet field, $n_{i j} \in \mathbb{N}$, and we suppressed coefficients. It is clear that, after a bi-unitary diagonalization, the top Yukawa coupling will be given by

$$
y_{t}=\mathcal{O}(g)+\mathcal{O}\left(s^{n}\right)
$$

with some $n \in \mathbb{N}$. However, if the singlet scale and the exponents $n_{i j}$ in (18) are such as to give rise to realistic Yukawa couplings for the $u$ and $s$ quarks, this effect is expected to be negligible. In fact, as we shall see next, localization effects in the compact dimensions will generically have sizable impact on $y_{t}$, completely overwhelming the effect described in the present subsection. 


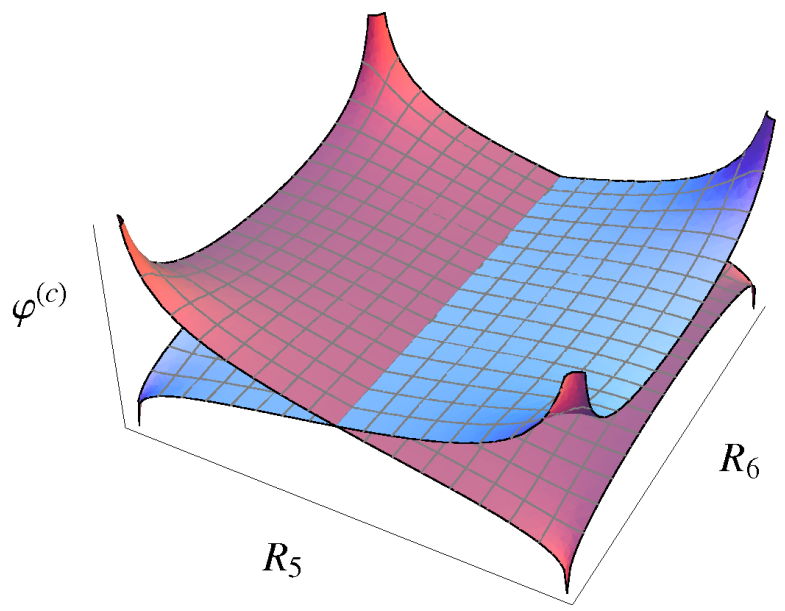

Figure 2: Localization of two wavefunctions $\varphi$ and $\varphi^{c}$ with opposite charges in the extra dimensions.

\subsection{Localization effects}

Building on earlier analyses of the 5D case [36,37], Lee, Nilles and Zucker (LNZ) [33] reported an interesting observation. Suppose there is a U(1) symmetry, called U(1) LNZ in what follows, with localized Fayet-Iliopoulos (FI) terms, i.e.

$$
\operatorname{tr}\left(q_{I}\right) \neq 0
$$

where the trace extends over the states localized at the fixed points, labeled by $I=$ $\left(n_{2}, n_{2}^{\prime}\right)$. It turns out that then bulk states with non-zero $\mathrm{U}(1)_{\mathrm{LNZ}}$ charge develop a non-trivial profile in the extra dimensions, cf. figure 2, In such a situation, the effective four-dimensional top Yukawa coupling, which is given by the overlap of the corresponding wave functions, generically gets reduced. It is amusing to see that the presence of fixed points, which break $N=2$ supersymmetry down to $N=1$, also allows us to discriminate between the $\varphi$ and $\varphi^{c}$ components of the hypermultiplet.

In [33] it was shown that the profile for the zero modes on the $6 \mathrm{D}$ orbifold $\mathbb{T}^{2} / \mathbb{Z}_{2}$ is given by

$$
\psi \simeq f \prod_{I}\left|\vartheta_{1}\left(\frac{z-z_{I}}{2 \pi} \mid \tau\right)\right|^{\frac{1}{2 \pi} g_{6} q_{\psi} \xi_{I}} \exp \left(-\frac{1}{8 \pi^{2} \tau_{2}} g_{6} q_{\psi} \xi_{I}\left(\operatorname{Im}\left(z-z_{I}\right)\right)^{2}\right),
$$

where we neglected a subleading logarithmic contribution, and $f$ is a normalization factor. We explicitly checked that this profile is the same for bosonic and fermionic degrees of freedom. The complex coordinate $z$ is defined as

$$
z=\frac{1}{R_{5}} x^{5}+\frac{\tau}{R_{6}} x^{6}
$$

with $\tau$ denoting the modular parameter of the torus. In our settings we assume the torus to be rectangular, $\tau=\mathrm{i} \tau_{2}=\mathrm{i} R_{6} / R_{5}$. The torus $\vartheta$-functions can be written as [38]

$$
\vartheta_{1}(z \mid \tau)=\sum_{n \in \mathbb{Z}} \mathrm{e}^{\mathrm{i} \pi \tau\left(n+\frac{1}{2}\right)^{2}+2 \pi \mathrm{i}\left(n+\frac{1}{2}\right)\left(z+\frac{1}{2}\right)}
$$


Furthermore,

$$
\xi_{I}=\frac{1}{16 \pi^{2}} g_{6} \Lambda^{2}\left(\frac{1}{4} \operatorname{tr}(q)+\operatorname{tr}\left(q_{I}\right)\right)
$$

is the quadratically divergent piece of the FI term with $q$ the charges of the bulk fields under the corresponding $\mathrm{U}(1), q_{I}$ the charges of fields localized at the fixed point $I$ and $\Lambda$ the cutoff of the theory. We will ignore the first term in the sum of the right-hand side of (24), i.e. we assume $\operatorname{tr} q=0$. We further take $\Lambda$ to be the higher-dimensional Planck scale, $\Lambda^{2}=M_{\mathrm{P}} / \sqrt{V_{56}}$ with $M_{\mathrm{P}}=2.43 \cdot 10^{18} \mathrm{GeV}$ and $V_{56}=2 \pi^{2} R_{5} R_{6}$ the volume of the extra dimensions. In addition we have the usual relation between the higher-dimensional gauge coupling and its four-dimensional counterpart, $g_{6}=\sqrt{V_{56}} g$. The constant $f$ can be determined through the normalization condition

$$
\int_{0}^{\pi R_{5}} \mathrm{~d} x_{5} \int_{0}^{2 \pi R_{6}} \mathrm{~d} x_{6}|\psi|^{2}=1
$$

In our case we consider the orbifold $\mathbb{T}^{2} / \mathbb{Z}_{2}$ with one Wilson line in the $y_{5}$ direction. This means that there are two pairs of equivalent fixed points, so all FI terms will have the same absolute value, given that the effective FI term in four dimensions vanishes. Then the lightest Kaluza-Klein masses are either $M=1 /\left(2 R_{5}\right)$ or $M=1 / R_{6}$, due to the presence of the Wilson line. Since we are interested in the limit $R_{5} \geq R_{6}$ and since the GUT group gets broken by the Wilson line, we identify the GUT scale with the corresponding Kaluza-Klein mass, $M_{\mathrm{GUT}}=1 /\left(2 R_{5}\right)$.

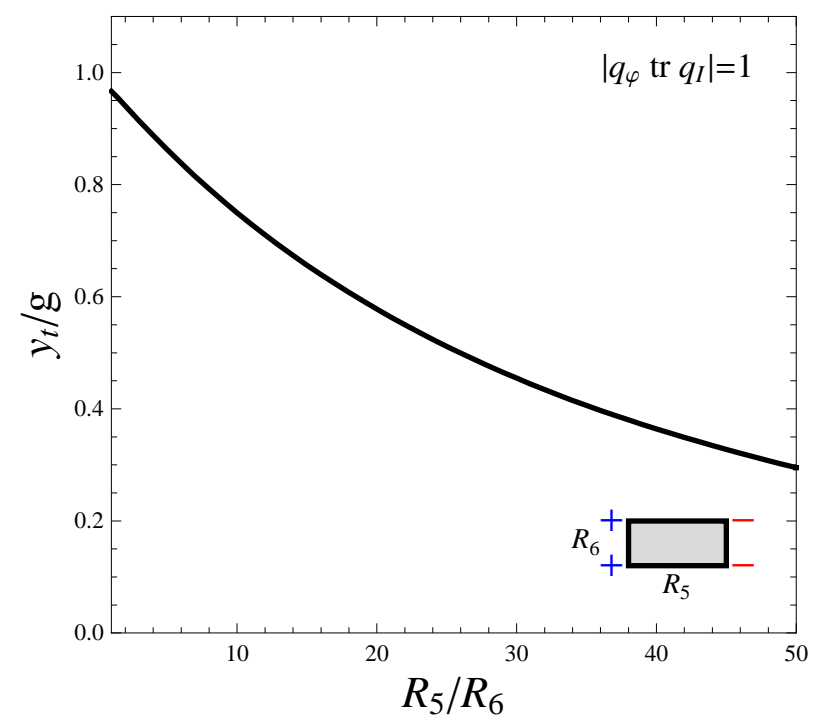

Figure 3: Suppression of the top Yukawa coupling $y_{t}$ relative to the gauge coupling $g$ due to the localization effects as a function of the anisotropy. The inlay indicates that the region $R_{5}>R_{6}$, where the distance between equivalent fixed points with coinciding traces is smaller that the one between inequivalent fixed points, is considered. In the other limit, i.e. for $R_{6} \gg R_{5}$, there is no analogous suppression.

Let us now briefly compare the terms giving rise to the top Yukawa and gauge couplings, respectively. While the first is proportional to the overlap integral over $h_{u} q_{3} \bar{u}_{3}$, 
the latter scales like the integral over $A \bar{u}_{3} \bar{u}_{3}^{\dagger}$. The Higgs field itself retains a flat profile since it comes from a non-Abelian gauge multiplet. Taking further into account that the profile of $\bar{u}_{3}^{\dagger}$ is identical to the one of $\bar{u}_{3}$, while the profiles of $q_{3}$ and $\bar{u}_{3}$ are inverse to each other, it is clear that the top Yukawa coupling will always be reduced with respect to the gauge coupling, when the correct normalization is taken into account. This effect is illustrated in figure 3. As can be seen, the reduction depends on the given charges and is more pronounced for anisotropic compactifications. This behavior is to be expected, since in the limit $R_{6} \rightarrow 0$ the $5 \mathrm{D}$ case should be recovered, where the bulk fields effectively become brane fields and hence the overlap should vanish. This is because in this limit the bulk fields get exponentially localized towards the opposite ends of the interval [36], such that the overlap becomes exponentially suppressed.

\section{Explicit string theory realization}

Our analysis is motivated by recent progress in string model building [23-28], where $\mathcal{O}(100)$ explicit (and globally consistent) models with the exact spectra of the MSSM, the so-called 'heterotic mini-landscape', have been derived. In a subclass of these models the top (but neither the bottom nor the $\tau$ ) Yukawa coupling is related to the gauge coupling. This applies in particular to the two models that have been analyzed in some detail, [23, 24, 26, 28] and the 'benchmark model 1A' in [27]. In what follows, we will study gauge-top unification in these models. Our analysis should be viewed as the first step towards a full string theory calculation, where one computes the FI term in string theory, takes into account arbitrary values for the torus parameter $\tau$ and the full volume of the six compact dimensions. In the present study, we will restrict ourselves to the somewhat naive orbifold GUT picture, and will take the cutoff $\Lambda$ to be the 6D Planck scale, as before.

\subsection{Motivation of the orbifold GUT picture}

Taking the orbifold GUT limit of a string compactification has a rather profound motivation. Witten proposed in a footnote [39, footnote 3] a possible way to explain the discrepancy between the string and the GUT scales. This can be accomplished by considering highly anisotropic compactifications, where $M_{\mathrm{GUT}}$ is associated to the inverse of the largest radius, while all (or most of) the other radii are much smaller. In this case, the volume of compact space can be small enough to ensure that the perturbative description of the setting is still appropriate. This idea has been studied in some detail more recently [40]. The outcome of the analysis is that the above puzzle can be resolved if the largest radius is by a factor 50 or so larger than the other radii. The question of how to stabilize the largest radius has also been addressed in the framework of field theory, and it has been found that one can indeed obtain $R_{5} \simeq 1 /\left(2 M_{\mathrm{GUT}}\right)$ [41-43]. Also here 
localized FI terms can play an important role. The question why one radius behaves so differently from the others will be addressed elsewhere [44]. In what follows, we will build on these results, and consider 6D orbifold GUT limits with particular emphasis on the highly anisotropic case $R_{5} \gg R_{6}$. The transverse four dimensions will not be considered.

\subsection{A specific example}

For concreteness, let us first focus on the benchmark model $1 \mathrm{~A}$ of [27]. In order to be in accord with the discussion in section 3.3 (and the analysis by LNZ [33]), here and below we take only $\mathrm{U}(1)$ factors orthogonal to the so-called 'anomalous U(1)' direction $t_{\text {anom }}$ into account. The general case will be explored elsewhere. One can choose the basis of these $\mathrm{U}(1) \mathrm{s}$ such that the local FI terms at the fixed points are entirely in one $\mathrm{U}(1)$ direction, called $\mathrm{U}(1)_{\mathrm{LNZ}}$ in what follows. The corresponding generator reads

$$
\mathrm{t}_{\mathrm{LNZ}}=\frac{1}{\sqrt{105}}\left(-\frac{3}{2}, 0,0,0,0,0,0,0\right)\left(-\frac{11}{4}, \frac{21}{4}, 0, \frac{11}{4}, 0, \frac{11}{4}, 0,0\right)
$$

and is normalized such that $\left|t_{\mathrm{LNZ}}\right|^{2}=1 / 2$. In this normalization, the charges of $\varphi$ and $\varphi^{c}$, containing $q_{3}$ and $\bar{u}_{3}$, are

$$
q_{\varphi}=-q_{\varphi^{c}}=\frac{1}{\sqrt{105}} \frac{3}{4}
$$

respectively. One further has

$$
\operatorname{tr}_{\mathrm{SU}(5) \text { brane }} q_{\mathrm{LNZ}}=\frac{64}{\sqrt{105}} \quad \text { and } \quad \operatorname{tr}_{\mathrm{SU}(4) \times \mathrm{SU}(2) \text { brane }} q_{\mathrm{LNZ}}=-\frac{64}{\sqrt{105}}
$$

where the $\mathrm{SU}(5)$ and $\mathrm{SU}(4) \times \mathrm{SU}(2)$ branes comprise the fixed points with $n_{2}=0$ and 1 , respectively, with the fixed points carrying half of the trace. We can hence apply the results of section 3.3 in order to evaluate $y_{t}$. The ratio $y_{t} / g$ for the present example is shown in figure 4. For this figure, we used our field theoretic estimate of the FI term, i.e. chose to identify $\Lambda$ with the $6 \mathrm{D}$ Planck scale, as before. As discussed in section 3.3 , the localization effect becomes more pronounced when the ratio $R_{5} / R_{6}$ increases. We also show what the suppression would be if we chose an increased cut-off, $\Lambda^{2}=4 M_{\mathrm{P}} / \sqrt{V_{56}}$, designed in such a way that for $R_{5}=50 R_{\geq 5} \Lambda$ equals the heterotic string scale, $M_{\text {string }} \simeq$ $8 \cdot 10^{17} \mathrm{GeV}$, as in the proposal discussed by Hebecker and Trapletti [40].

It is amazing to see that the top Yukawa coupling gets only reduced if we take the 'more appealing' orbifold GUT limit $R_{5} \gg R_{6}$. If we were to choose $R_{6} \gg R_{5}$, which would lead us to an orbifold GUT with $\mathrm{SU}(3) \times \mathrm{SU}(3)$ bulk group that gets broken to the SM at the two equivalent boundaries, the top Yukawa coupling would no longer be suppressed since $\operatorname{tr} q_{I}$ vanishes at both boundaries. 


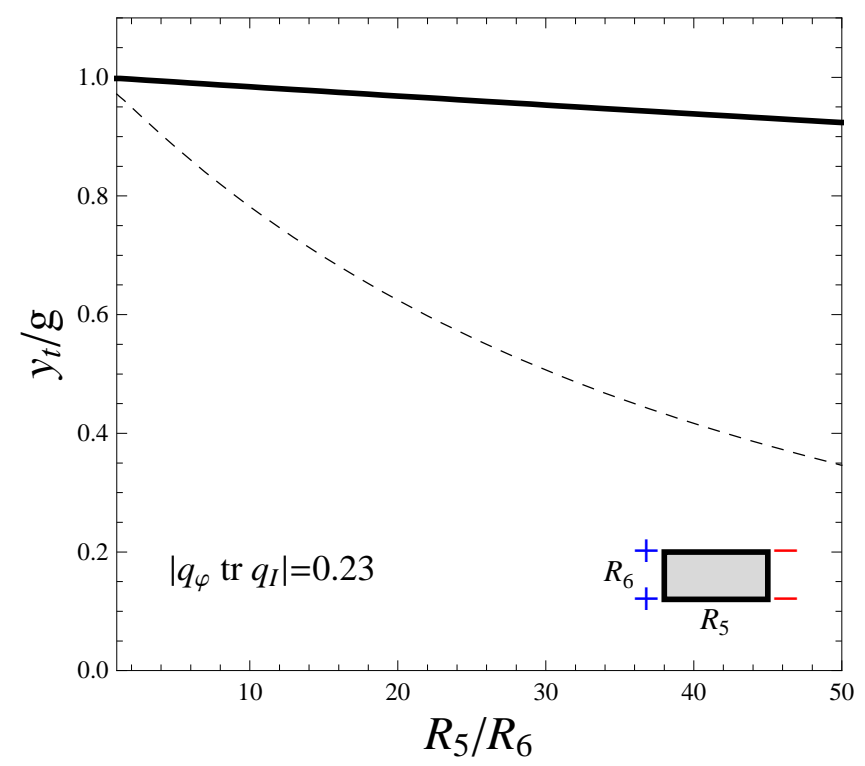

Figure 4: Estimate of $y_{t} / g$ in the benchmark model 1A of [27]. For comparison, the dashed line shows the suppression which occurs with an increased cut-off, $\Lambda \rightarrow 2 \Lambda$.

\subsection{Mini-landscape survey}

We have repeated the analysis of section 4.2 for a subclass of the heterotic mini-landscape models [25,27], in which

- there is an orbifold GUT limit as described in section 2,

- it has been explicitly verified that exotics decouple consistently with supersymmetry, i.e. with vanishing $F$ - and $D$-terms.

All these models, including the model derived in $[23,24,26,28]$ and the 'benchmark model 1A' discussed before, are based on the same shift vector. They turn out to have the following family structure (up to vector-like states):

- $1^{\text {st }}$ and $2^{\text {nd }}$ families come from 16-plets localized at $\mathrm{SO}(10)$ fixed points, which correspond to the fixed points with $n_{2}=0$ in the orbifold GUT limit (figure 1);

- $3^{\text {rd }}$ family $\bar{d}$ and $\ell$ (i.e. the $3^{\text {rd }}$ family $\overline{5}$ in $\mathrm{SU}(5)$ language) come from the $T_{2 / 4}$ twisted sectors and therefore are localized on two-dimensional submanifolds in compact $6 \mathrm{D}$ space;

- $3^{\text {rd }}$ family $\bar{u}, \bar{e}$ and $q$ as well as the Higgs fields $h_{u}$ and $h_{d}$ are bulk fields, i.e. free to propagate everywhere in compact space.

Only 4 out of 56 candidate models do not have localized FI terms.

Yukawa couplings connecting the Higgs fields to matter may be written as overlap integrals. One could then expect that the couplings of the first two generations are 
suppressed by the total 6D volume while the $\tau$ and $b$ Yukawas, $y_{\tau}$ and $y_{b}$, are suppressed by the volume of the $4 \mathrm{D}$ space transverse to the two-dimensional submanifold, while the top Yukawa $y_{t}$ is unsuppressed, as discussed before. This leads us to the hierarchy

Yukawa couplings of the first two generations $\ll y_{\tau}, y_{b} \ll y_{t}$.

It should be mentioned that these localization properties are highly non-trivial (and come out 'for free'). In fact, the cancellation of all 4D and higher-dimensional gauge anomalies, which is guaranteed by modular invariance [45], appears rather miraculous in field theory [26]. Also discrete anomalies have been shown to cancel [46].

Let us now come back to the question of the value of $y_{t}$ at the compactification or GUT scale. As before in section 4.2, it is possible to rotate the basis of $U(1)$ factors (orthogonal to $t_{\text {anom }}$ ) such that there is precisely one $U(1)$, which we again call $U(1)_{\mathrm{LNZ}}$, with the following properties:

- the chiral multiplets $\varphi$ and $\varphi^{c}$ have non-zero (and opposite) U(1) LNZ charges;

- the traces $\operatorname{tr}_{\mathrm{SU}(5) \text { brane }} q_{\mathrm{LNZ}}$ and $\operatorname{tr}_{\mathrm{SU}(4) \times \mathrm{SU}(2)}$ brane $q_{\mathrm{LNZ}}$ have opposite sign (as in $(28))$;

- $\left|\mathrm{t}_{\mathrm{LNZ}}\right|^{2}=\frac{1}{2}$, i.e. the charges are in "GUT normalization".

The statistics of the localized traces and the charges of the bulk hypermultiplets $\varphi$ and $\varphi^{c}$ are depicted in figure 5 .

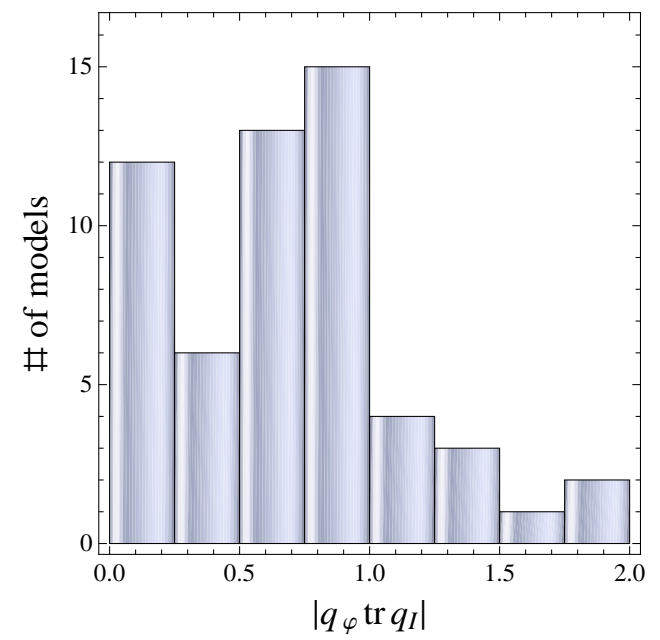

(a) $\left|q_{\varphi} \operatorname{tr} q_{I}\right|$ at $I=(0,0)$.

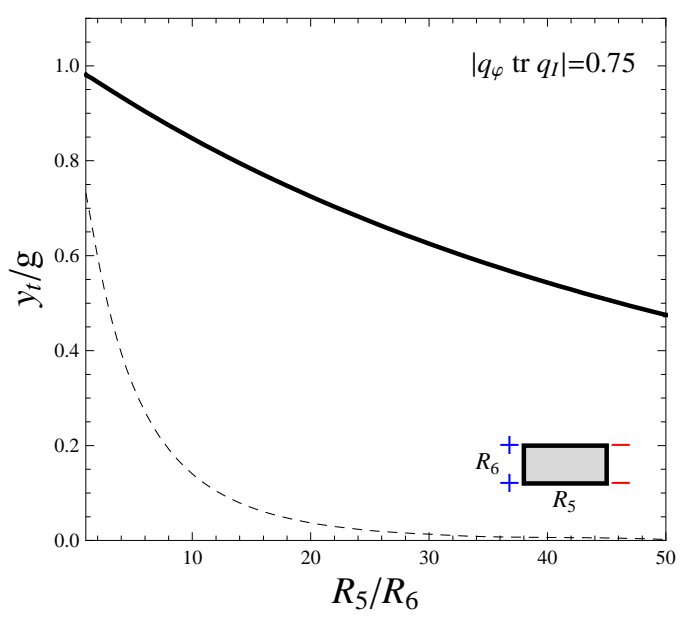

(b) $y_{t} / g$.

Figure 5: Statistics of $\left|q_{\varphi} \operatorname{tr} q_{\mathrm{LNZ}}\right|_{n_{2}=n_{2}^{\prime}=0} \mid$ in a class of string-derived orbifold GUTs and suppression of the top Yukawa coupling for the average value. 


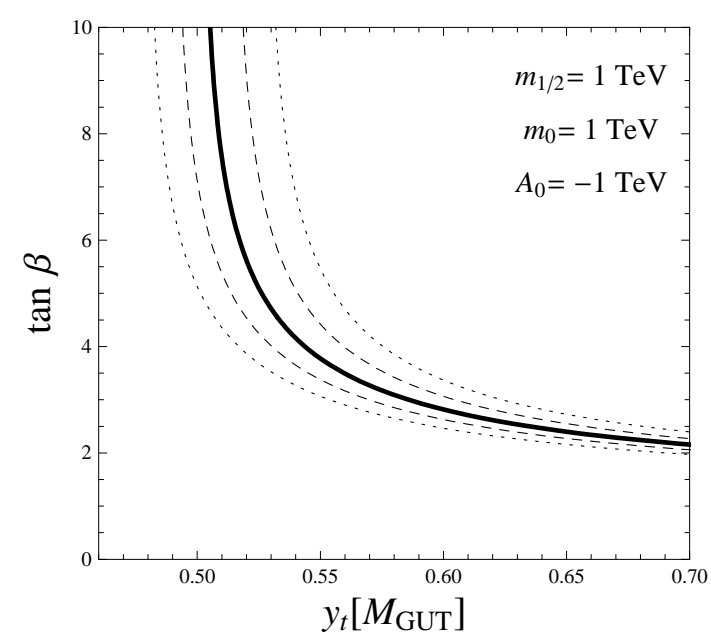

(a) $y_{t}$ vs. $\tan \beta$.

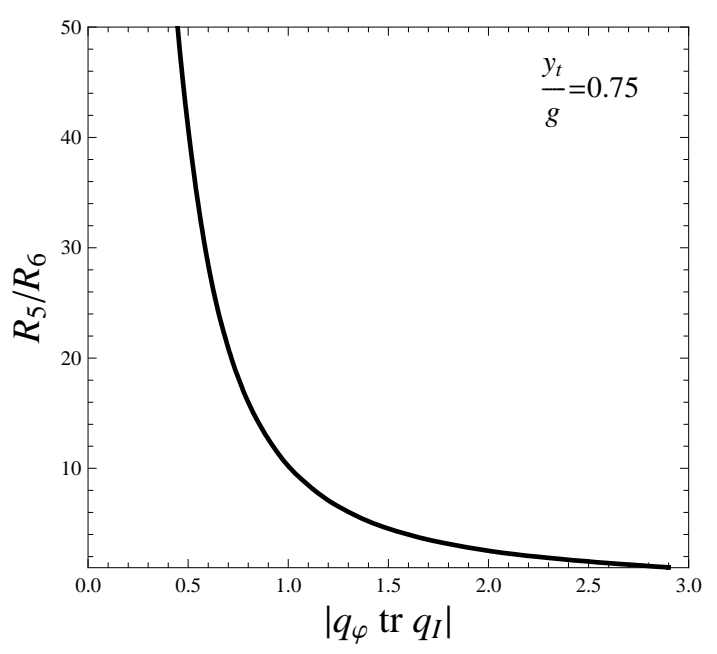

(b) $R_{5} / R_{6}$ vs. $\left|q_{\varphi} \operatorname{tr} q_{I}\right|$.

Figure 6: (a) $\tan \beta$ as a function of the top Yukawa coupling at the GUT scale. The central line corresponds to the central value of the top quark mass, whereas the dashed and dotted lines correspond to the one and two sigma intervals, respectively. A larger top mass results in a larger $\tan \beta$. (b) shows for given $q_{\varphi} \operatorname{tr} q_{I}$ the anisotropy that is needed to reduce $y_{t} / g$ to 0.75 .

\section{Phenomenological implications}

Let us now come to some phenomenological implications of the gauge-top unification scenario. Given a certain pattern of soft terms as well as the top Yukawa coupling at the GUT scale, the value of $\tan \beta$ can be determined. Note that the value of $\tan \beta$ is also quite sensitive to the mass of the top quark, where the latest experimental value is given by $m_{t}=173.1 \pm 1.3 \mathrm{GeV}[32]$.

It turns out that the resulting value for $\tan \beta$ mainly depends on $y_{t}\left(M_{\mathrm{GUT}}\right)$. To illustrate this dependence we plot the top Yukawa coupling at the GUT scale versus $\tan \beta$ for different values of the top quark mass in figure 6. We impose a specific set of boundary conditions at the GUT scale, the so-called mSUGRA set with $m_{0}=m_{1 / 2}=-A_{0}=1 \mathrm{TeV}$, and use SOFTSUSY [47] for our numerical analysis.

The value of the unified gauge coupling on the other hand turns out to be only very weakly dependent on the soft parameters and is always close to $g \simeq 0$. This implies that, given the tree-level relation $y_{t}=g \simeq 0.7$, the resulting value for $\tan \beta$ is quite small, $\tan \beta \simeq 2$. An immediate question is whether such small values for $\tan \beta$ and hence the tree-level relation are still valid, since the small $\tan \beta$ region is probed experimentally by searches for the light Higgs boson [48]. This tension can be seen by comparing the LEP bound on the SM Higgs mass, $m_{h} \geq 114.4 \mathrm{GeV}$, to the theoretical upper bound on the lightest Higgs mass as a function of $\tan \beta$. At tree-level the maximal mass for the lightest Higgs is given by $m_{h}^{2} \simeq M_{Z}^{2} \cos ^{2} 2 \beta$, which vanishes for $\tan \beta=1$. Radiative corrections 
can significantly increase the Higgs mass compared to the tree-level value, but still $m_{h}$ is minimized for $\tan \beta$ around one in the MSSM. While the LEP Higgs bound does not apply for all of the MSSM parameter space, in the small $\tan \beta$ region it is applicable to a good approximation, since here the lightest CP-even Higgs boson couples to the $Z$ with SM-like strength [49]. For the so called ' $m_{h}^{\max }$ scenario' within the MSSM, designed such that for fixed values of $m_{t}$ and $M_{\text {SUSY }}$ the predicted value of the lightest Higgs is maximized for each value of $\tan \beta$ and $m_{A}$, the lower bound on $\tan \beta$ is around 2 [49]. Although hence values of $\tan \beta$ around 2 are still possible, one should not forget that this is possible only when the parameters are tuned accordingly. In a large part of the parameter space $\tan \beta$ has to be larger. To see what is natural as the 'smallest value of $\tan \beta$ without too much tuning', we restrict ourselves to mSUGRA. Taking $m_{1 / 2}=m_{0}=-A_{0}=1 \mathrm{TeV}$ and the top mass at its two sigma upper bound, the predicted Higgs mass is above the LEP bound for $\tan \beta \gtrsim 3.3$. This in turn implies a 'natural range' for the top Yukawa coupling at the GUT scale of $0.48 \lesssim y_{t} \lesssim 0.6$, which translates into $0.69 \lesssim y_{t} / g \lesssim 0.86$. Note that in the presence of vector-like matter (in complete SU(5) representations) with masses below the GUT scale the relative suppression of $y_{t}$ has to be stronger.

In conclusion we can say that the naive picture of gauge-top unification, although not excluded, seems to be possible for very special patterns of the soft terms only. However, we have seen in the previous sections that the top Yukawa coupling gets somewhat reduced with respect to the tree-level relation $y_{t}=g$, depending on the geometry of the extra-dimensional space. Turning this around means that, given a value for $\tan \beta$, we gain access to the geometry of the extra-dimensional space. In particular it seems that highly anisotropic compactifications are favored, in accord with [40].

Our findings seem to give another motivation for highly anisotropic string compactifications. The top Yukawa coupling seems to give a preference to this limit.

\section{Discussion}

We have discussed orbifold GUT scenarios in which the top Yukawa coupling arises from gauge interactions. This leads to the tree-level relation $y_{t}=g$ at the GUT or compactification scale. It turns out that in scenarios with localized FI terms $y_{t}$ is suppressed against $g$, which seems also to be required by data. The suppression depends on the size of the localized FI terms, the charge of the bulk hypermultiplets and, in particular, on the geometry of compact space. We have analyzed string-derived models which reproduce the MSSM below the compactification scale and which possess orbifold GUT limits with the above features. This allowed us to get a feeling for what the FI terms and charges 'should be'. Using this as an input, we find that the observed value of the top Yukawa coupling seems to favor anisotropic geometries, which might also be a key ingredient for reconciling the scale of grand unification with the Planck or string scales. This result 
might be interpreted as further support for the idea of orbifold GUTs and, in particular, for their embedding in the heterotic string.

As mentioned, our analysis should be viewed as a step towards getting sufficiently accurate predictions from string theory. The precision of the present analysis is mainly limited by our ignorance of the cut-off $\Lambda$, for which we simply used the 6D Planck scale. One can improve the accuracy significantly by directly computing the FI terms in string theory. Another important question is why, from a top-down perspective, one radius behaves so differently from the others. We plan to clarify these issues in the near future.

\section{Acknowledgments}

We would like to thank H.P. Nilles, P.K.S. Vaudrevange and M. Winkler for useful discussions and H.M. Lee for correspondence. This research was supported by the DFG cluster of excellence Origin and Structure of the Universe, and the SFB-Transregio 27 "Neutrinos and Beyond" by Deutsche Forschungsgemeinschaft (DFG). The work of P.H. was supported by the Alexander von Humboldt foundation.

\section{References}

[1] H. Georgi and S. L. Glashow, Phys. Rev. Lett. 32 (1974), 438-441.

[2] H. Fritzsch and P. Minkowski, Ann. Phys. 93 (1975), 193-266.

[3] L. J. Dixon, J. A. Harvey, C. Vafa, and E. Witten, Nucl. Phys. B261 (1985), 678686.

[4] L. J. Dixon, J. A. Harvey, C. Vafa, and E. Witten, Nucl. Phys. B274 (1986), 285314.

[5] L. E. Ibáñez, H. P. Nilles, and F. Quevedo, Phys. Lett. B187 (1987), 25-32.

[6] L. E. Ibáñez, J. E. Kim, H. P. Nilles, and F. Quevedo, Phys. Lett. B191 (1987), 282-286.

[7] J. A. Casas, E. K. Katehou, and C. Muñoz, Nucl. Phys. B317 (1989), 171.

[8] J. A. Casas and C. Muñoz, Phys. Lett. B214 (1988), 63.

[9] A. Font, L. E. Ibáñez, H. P. Nilles, and F. Quevedo, Nucl. Phys. B307 (1988), 109, Erratum ibid. B310.

[10] A. Font, L. E. Ibáñez, F. Quevedo, and A. Sierra, Nucl. Phys. B331 (1990), 421-474.

[11] Y. Kawamura, Prog. Theor. Phys. 103 (2000), 613-619, [hep-ph/9902423]. 
[12] Y. Kawamura, Prog. Theor. Phys. 105 (2001), 999-1006, [hep-ph/0012125].

[13] G. Altarelli and F. Feruglio, Phys. Lett. B511 (2001), 257-264, [hep-ph/0102301].

[14] L. J. Hall and Y. Nomura, Phys. Rev. D64 (2001), 055003, [hep-ph/0103125].

[15] A. Hebecker and J. March-Russell, Nucl. Phys. B613 (2001), 3-16, [hep$\mathrm{ph} / 0106166]$.

[16] T. Asaka, W. Buchmüller, and L. Covi, Phys. Lett. B523 (2001), 199-204, [hep$\mathrm{ph} / 0108021]$.

[17] L. J. Hall, Y. Nomura, T. Okui, and D. R. Smith, Phys. Rev. D65 (2002), 035008, [hep-ph/0108071].

[18] G. Burdman and Y. Nomura, Nucl. Phys. B656 (2003), 3-22, [hep-ph/0210257].

[19] T. Kobayashi, S. Raby, and R.-J. Zhang, Phys. Lett. B593 (2004), 262-270, [hep$\mathrm{ph} / 0403065]$.

[20] S. Förste, H. P. Nilles, P. K. S. Vaudrevange, and A. Wingerter, Phys. Rev. D70 (2004), 106008, [hep-th/0406208].

[21] T. Kobayashi, S. Raby, and R.-J. Zhang, Nucl. Phys. B704 (2005), 3-55, [hep$\mathrm{ph} / 0409098]$.

[22] W. Buchmüller, K. Hamaguchi, O. Lebedev, and M. Ratz, Nucl. Phys. B712 (2005), 139-156, [hep-ph/0412318].

[23] W. Buchmüller, K. Hamaguchi, O. Lebedev, and M. Ratz, Phys. Rev. Lett. 96 (2006), 121602, [hep-ph/0511035].

[24] W. Buchmüller, K. Hamaguchi, O. Lebedev, and M. Ratz, Nucl. Phys. B785 (2007), 149-209, [hep-th/0606187].

[25] O. Lebedev, H. P. Nilles, S. Raby, S. Ramos-Sánchez, M. Ratz, P. K. S. Vaudrevange, and A. Wingerter, Phys. Lett. B645 (2007), 88, [hep-th/0611095].

[26] W. Buchmüller, C. Lüdeling, and J. Schmidt, JHEP 09 (2007), 113, [arXiv:0707.1651 [hep-ph]].

[27] O. Lebedev, H. P. Nilles, S. Raby, S. Ramos-Sánchez, M. Ratz, P. K. S. Vaudrevange, and A. Wingerter, Phys. Rev. D77 (2007), 046013, [arXiv:0708.2691 [hep-th]].

[28] W. Buchmüller and J. Schmidt, Nucl. Phys. B807 (2009), 265-289, [0807.1046].

[29] M. Ratz, (2007), arXiv:0711.1582 [hep-ph]. 
[30] H. P. Nilles, S. Ramos-Sánchez, M. Ratz, and P. K. S. Vaudrevange, Eur. Phys. J. C59 (2009), 249-267, [0806.3905].

[31] I. Gogoladze, Y. Mimura, and S. Nandi, Phys. Lett. B562 (2003), 307-315, [hep$\mathrm{ph} / 0302176]$.

[32] Tevatron Electroweak Working Group, (2009), 0903.2503.

[33] H. M. Lee, H. P. Nilles, and M. Zucker, Nucl. Phys. B680 (2004), 177-198, [hepth/0309195].

[34] N. Arkani-Hamed, T. Gregoire, and J. Wacker, JHEP 03 (2002), 055, [hepth/0101233].

[35] H. Georgi, (1974), in: Particles and Fields 1974, ed. C. E. Carlson (AIP, NY, 1975) p. 575 .

[36] S. Groot Nibbelink, H. P. Nilles, and M. Olechowski, Nucl. Phys. B640 (2002), 171-201, [hep-th/0205012].

[37] S. Groot Nibbelink, H. P. Nilles, M. Olechowski, and M. G. A. Walter, Nucl. Phys. B665 (2003), 236-272, [hep-th/0303101].

[38] D. Mumford, Tata lectures on theta I, 1. ed., Birkhäuser, 1983.

[39] E. Witten, Nucl. Phys. B471 (1996), 135-158, [hep-th/9602070].

[40] A. Hebecker and M. Trapletti, Nucl. Phys. B713 (2005), 173-203, [hep-th/0411131].

[41] W. Buchmüller, R. Catena, and K. Schmidt-Hoberg, Nucl. Phys. B804 (2008), 7089, [0803.4501].

[42] C. Gross and A. Hebecker, (2008), 0812.4267.

[43] W. Buchmüller, R. Catena, and K. Schmidt-Hoberg, (2009), 0902.4512.

[44] R. Kappl, S. Ramos-Sánchez, M. Ratz, and P. K. S. Vaudrevange, (2009), in preparation.

[45] C. Vafa, Nucl. Phys. B273 (1986), 592.

[46] T. Araki et al., Nucl. Phys. B805 (2008), 124-147, [0805.0207].

[47] B. C. Allanach, Comput. Phys. Commun. 143 (2002), 305-331, [hep-ph/0104145].

[48] S. Heinemeyer, W. Hollik, and G. Weiglein, JHEP 06 (2000), 009, [hep-ph/9909540].

[49] G. Degrassi, S. Heinemeyer, W. Hollik, P. Slavich, and G. Weiglein, Eur. Phys. J. C28 (2003), 133-143, [hep-ph/0212020]. 